\title{
HAND GESTURE RECOGNITION USING ULTRASONIC SENSOR AND ATMEGA128 MICROCONTROLLER
}

\author{
Nidhi Gupta ${ }^{1}$, Ramandeep Singh ${ }^{2}$, Sidharth Bhatia ${ }^{3}$ \\ ${ }^{1} P G$ Student, EECE Department, ITM University, Gurgaon, Haryana, India \\ ${ }^{2}$ Assistant Professor, EECE Department, ITM University, Gurgaon, Haryana, India \\ ${ }^{3}$ Assistant Professor, EECE Department, ITM University, Gurgaon, Haryana, India
}

\begin{abstract}
The aim of this paper is to present a system that detects the hand gesture motions using the principle of Doppler Effect. Ultrasonic waves are transmitted by a sensor module and are reflected by a moving hand. The received waves are frequency shifted due to Doppler Effect. These frequency shifts in the ultrasonic waves are characterized to determine gestures. The gestures once recognized are mapped into commands to control the movement of a small robot. Current research work spans only four gestures: front movement, back movement, move left and move right.
\end{abstract}

Keywords: Hand Gesture Recognition, Ultrasonic Doppler, Human Computer Interface, Sonar, Presence Detection

\section{INTRODUCTION}

In order to recognize hand gestures, the principle of Doppler Effect is used here. An ultrasonic wave of frequency 22 $\mathrm{KHz}$ is generated with the help of an ultrasonic sensor. When a hand is waved near the source of Ultrasound wave, there is shift in the frequency of the sound wave, due to Doppler Effect. The received signal is then analyzed for characteristic shifts to determine whether the motion was push, pull, clockwise rotation or anti-clockwise rotation. The results will be indicated by the movement of a small robot. The robot will move towards forwards, if the motion of the hand was push; backwards if the hand motion was push; left if the hand motion was a clockwise rotation; and right if the hand motion was an anti-clockwise rotation.

This project is based on the Microsoft's SoundWave [1] and Acoustic Doppler Sonar (ADS) [2].

The system comprises of an ultrasonic pair of transmitter and a receiver. The transmitter transmits an inaudible tone that is reflected by a hand in motion, in proximity. The reflected tone undergoes a frequency shift due to Doppler Effect. This amount of shift is dependent on the velocity of hand. The receiver captures the frequency shifted signals. The signals received are used to characterize the movement of the hand, in time domain.

The incoming time-domain signal are buffered [3], and Fourier transform is applied on them. The result of this operation is magnitude vectors that are spread equally over the spectral width.

The relationship between observed frequency $f$ and emitted frequency $\mathrm{f}_{0}$ is given by

$$
f=\left(\frac{c+v_{r}}{c+v_{s}}\right) f_{0}
$$

Where,

$\mathrm{c}$ is the velocity of waves in the air;

$\mathrm{v}_{\mathrm{r}}$ is the velocity of the receiver in air; if the object is moving towards the source then positive (and negative in the other direction);

$\mathrm{v}_{\mathrm{s}}$ is the velocity of the source in air; if the source is moving away from the receiver then positive (negative if moving towards)

After each FFT vector is computed, it is further processed to determine the bandwidth of the signals, speed of gestures and motion detection. The detected motions are then converted to robotic commands.

\section{BLOCK DIAGRAM}

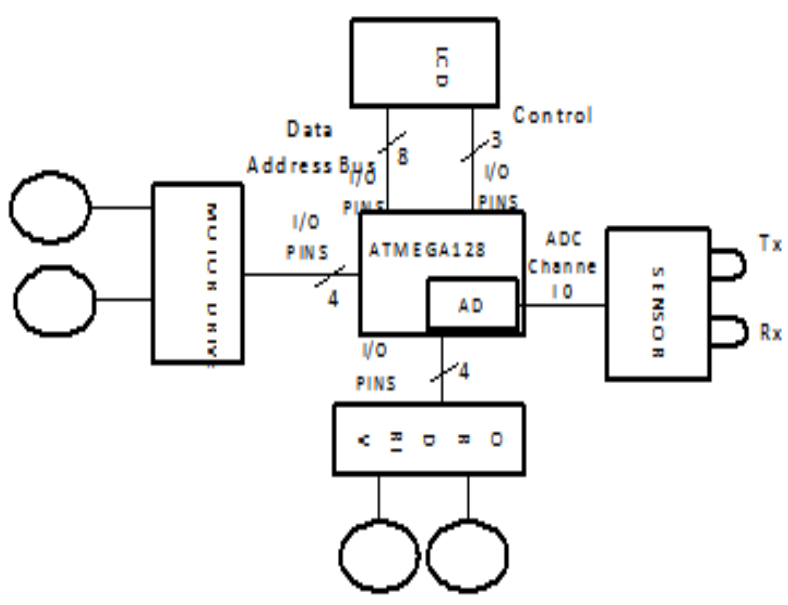

Fig 1: Components in the hardware setup

The hardware configuration for this system is as follows: An ultrasonic module has on board transmitter and receiver. This module is connected to the ATMEGA128 
microcontroller. The analog input received is converted to digital values by the inbuilt ADC (Analog to digital converter) of the microcontroller.

The microcontroller (AVR ATMEGA128) emits the waves at a regular interval. If there is any obstacle (or hand, in this case) then the ultrasonic waves will be reflected, frequency shifted. The microcontroller performs a multiplication function that to compare the incoming and outgoing signals in hardware. This multiplier function uses the fundamental property of sine waves to find the difference in frequency between incoming and outgoing signals in hardware. The product of two sine waves produces two phase-shifted sine waves, one with a frequency that is the difference in frequencies between that of the two input waves, and the other with a frequency that is the sum of the two input waves:

$\sin \left(2 \pi f_{a} x\right) \sin \left(2 \pi f_{b} x\right)=$

$\cos \left(2 \pi\left(f_{a}-f_{b}\right) x\right)+\cos \left(2 \pi\left(f_{a}+f_{b}\right) x\right) / 2$

The robot will receive commands from the microcontroller corresponding to each gesture.

LCD display is used to provide textual information of the signal analyzed.

The following are the gestures and corresponding functions that are executed in the current project:

Table 1: Gesture and function chart

\begin{tabular}{|l|l|}
\hline Gesture Motion & Function \\
\hline Front Movement & Move Forward \\
\hline Back Movement & Move Backward \\
\hline $\begin{array}{l}\text { Anti- Clockwise } \\
\text { Movement }\end{array}$ & Move Left \\
\hline Clockwise Movement & Move Right \\
\hline
\end{tabular}

\section{ALGORITHM}

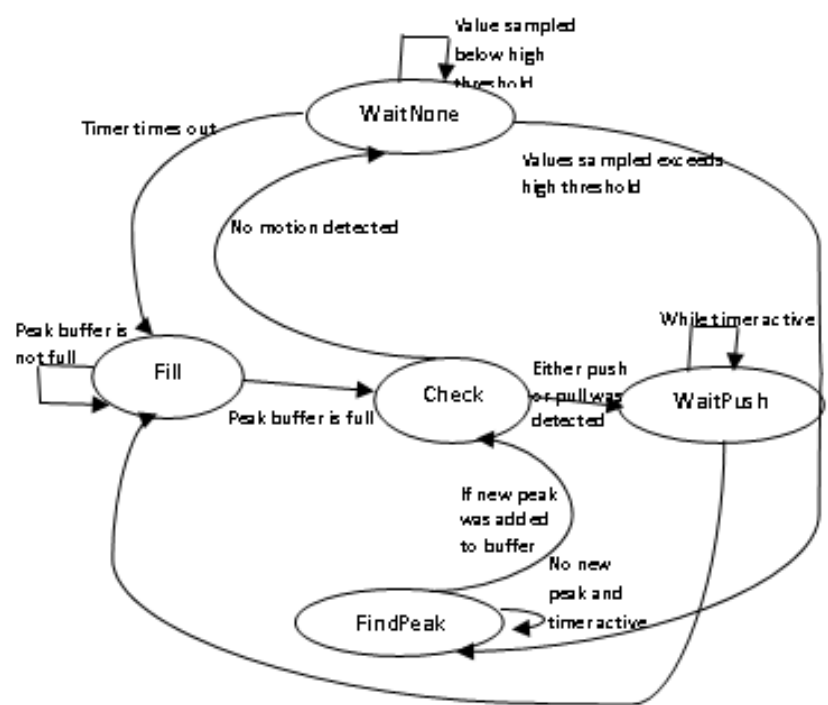

Fig 2: Finite State Machine
Initial state is the Fill State. The peaks (local highs above a predefined threshold) in the ADC samples fill the buffer until full. Check state is where the local highs are analyzed to determine if the motion is one of the six gestures predefined in the database. The total number of peaks that dropped from HIGH to LOW, or number of peaks that rose from LOW to HIGH are counted. WaitPush is transited to from Check state if there is any gesture in the sample data collected. WaitNone is transited to from Check State if there was no gesture. FindPeak fills a buffer with one peak, as against the Fill state where the buffer is completely filled with local HIGHs. This separate state is required because the buffer will not need to be completely refilled even if no gesture was detected.

\section{HARDWARE CONSIDERATION}

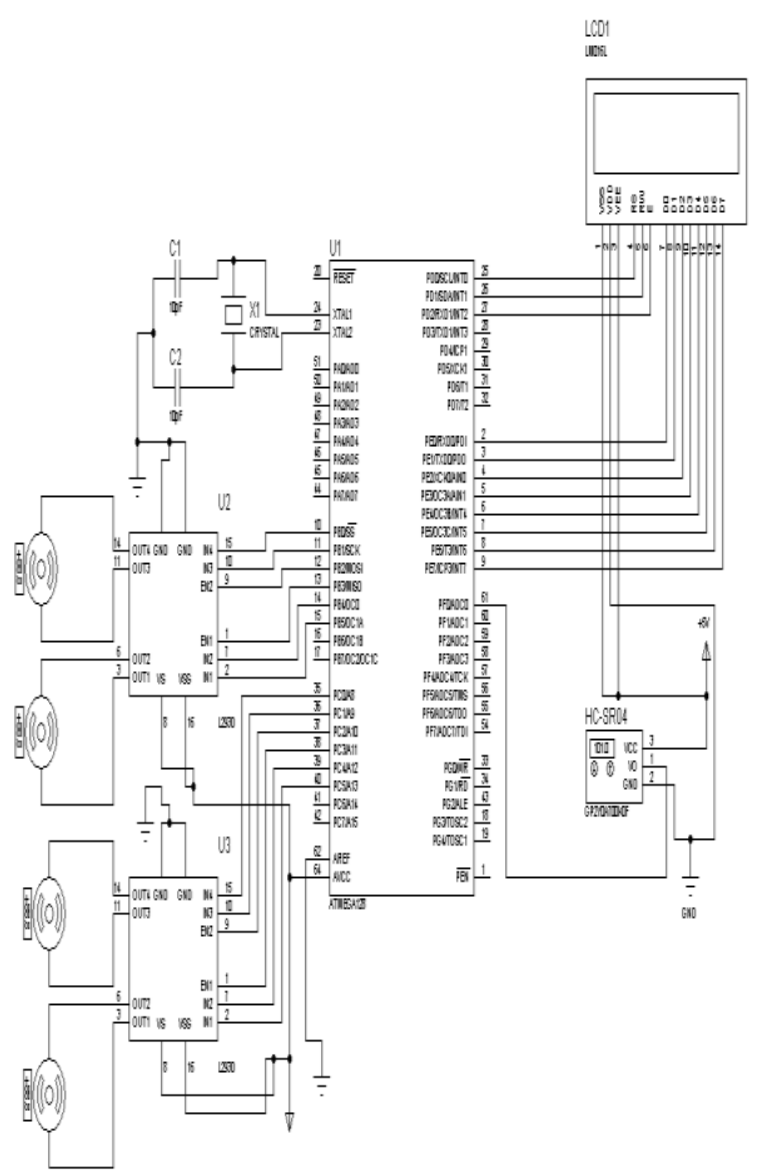

Fig 3: Schematic diagram for hand gesture controlled robotic movement

The above circuit comprises of ATMEGA128 microcontroller, HC-SR04 Ultrasonic sensor, two motor driver ICs L293, and four DC motors. The sensor has onboard transmitter and receiver. The sensor's TRIG and ECHO pins are connected to the microcontroller's ADC port. The motor driver is connected to the other input/output port.

The microcontroller gives a logic HIGH at the pin connected to TRIG of sensor at regular intervals. The ultrasonic waves are emitted and if there is any object obstructing these 
signals, then the ultrasonic waves are reflected back. The receiver in the ultrasonic module receives the frequency shifted signal at the pin connected to ECHO of sensor. These received waves are buffered and stored in the microcontroller. The microcontroller analyzes the signal for a pattern. If the pattern matches a gesture in the database, then it further determines the command associated with the gesture. The command is given in the form of logic HIGH or LOW to the pins of the motor driver L293D. These commands correspond to the robotic movements, as mentioned in Table 1.

The processing of the microcontroller is also reflected in the LCD. The gestures determined and the corresponding robotic actions are displayed on the Liquid Crystal Display, simultaneously as the commands are given to the robot. This provides an additional result display as well as verification mechanism.

Table 2: PIN Description

\begin{tabular}{|c|c|c|c|}
\hline $\begin{array}{l}\text { PIN } \\
\text { NUMBER }\end{array}$ & NAME & I/O & FUNCTION \\
\hline 61 & PORTF.0 & IN & $\begin{array}{l}\text { Receive input } \\
\text { from the sensor }\end{array}$ \\
\hline $\begin{array}{l}12, \quad 13,37, \\
38\end{array}$ & $\begin{array}{l}\text { PORTB. } 2, \\
\text { PORTB. } 3, \\
\text { PORTC. } 2, \\
\text { PORTC. } 3\end{array}$ & OUT & Enable the motor \\
\hline $\begin{array}{ll}10,11, & 14, \\
15,35, & 36, \\
39,40 & \end{array}$ & $\begin{array}{l}\text { PORTB.0, } \\
\text { PORTB. } 1, \\
\text { PORTB.4, } \\
\text { PORTB. } 5 \text {, } \\
\text { PORTC. } 0, \\
\text { PORTC. } 1, \\
\text { PORTC. } 4, \\
\text { PORTC. } 5\end{array}$ & OUT & DC motor values \\
\hline 25 & PORTD.0 & OUT & LCD RS pin \\
\hline 26 & PORTD.1 & OUT & LCD RW pin \\
\hline 27 & PORTD.2 & OUT & LCD EN pin \\
\hline $2-9$ & PORTE & OUT & LCD DATA \\
\hline
\end{tabular}

Table 2 (contd.): PIN Description

\begin{tabular}{|l|l|l|l|}
\hline $\begin{array}{l}\text { PIN } \\
\text { NUMBER }\end{array}$ & NAME & I/O & FUNCTION \\
\hline $23 / 24$ & XTAL & IN & $\begin{array}{l}\text { Crystal } \\
\text { Oscillator }\end{array}$ \\
\hline 64 & Vcc & IN & Power source \\
\hline 62 & ARef & IN & Ground \\
\hline
\end{tabular}

\section{APPLICATION AREAS}

Gesture recognition is useful in processing information from human beings that is not conveyed through speech or other methods. This technology is useful in following areas:

a. Immersive gaming technology: Gestures may be used to control interactions with the gaming console and give a more interactive and immersive experience.

b. Sign Language interpretation: Gesture recognition can be used to transcribe signs into text, just like speech recognition. This would be greatly helpful for the speech impaired.

c. Control through facial gestures: This technology can be used for applications with even more precision like recognizing face gestures. This will be helpful in situations when users cannot use other input interfaces like mouse or keyboard or even hand gestures. This would be additionally helpful in applications like mood sensing.

d. Alternative computer interfaces: Strong gesture recognition can be used to accomplish common tasks performed traditionally with the current input devices such as mouse or keyboard. Gestures, along with other methodologies like speech recognition can be made to control the electronic appliances and gadgets completely or with little need to type or touch. [4] [5] [6]

e. Remote control: By using gesture recognition, it is possible to use hand alone as a remote control for various devices. The signal must not only indicate the desired response, but also which device to be controlled. [9] [10] [11]

f. Home Appliances control: It is possible to extend the gesture recognition technology to control the household appliances. [10]

\section{ADVANTAGES \& LIMITATIONS}

Gesture recognition is very useful for automation. Gestures, a natural language of humans, provide an intuitive and effortless interface for communication with the computers. They will reduce our need for devices like mouse, keys, remote control or keys for interaction with the electronic devices. When combined with other advanced user interface technologies such as voice commands and face recognition, gestures can create a richer user experience that strives to understand the human "language," thereby fueling the next wave of electronic innovation.

This technology is limited in the sense that all whole of human signs or gestures are not recognizable using this technology. The ultrasonic waves spread out and cannot be used to detect gestures like victory sign, where the gesture is made by two fingers.

\section{RESULTS}

The gesture recognition using ultrasonic waves is found to be accurate and reliable. The methodology for testing comprised of movement of single hand or multiple hands. Single hand movement was detected accurately. When there are multiple hands, the movement is not detected accurately. The detection did not take into account the background area. The noise in human audible range did not affect the detection.

\section{CONCLUSIONS \& FUTURE SCOPE}

Additional gesture recognition opportunities exist in medical applications where, for health and safety reasons, a nurse or doctor may not be able to touch a display or track-pad but still needs to control a system. In other cases, the medical 
professional may not be within reach of the display yet still needs to manipulate the content being shown on the display. Appropriate gestures, such as hand swipes or using a finger as a virtual mouse, are a safer and faster way to control the device.

Gesture recognition may be used in automobiles for user control, and among other motivations as an incremental convenience and safety capability.

Robots, if trained to recognize some gestures can be used in situations of social needs, like rehabilitation or catastrophe, more independently.

\section{ACKNOWLEDGEMENTS}

Several people helped me through the project, notably the Lab Assistants, for providing hardware and support; my beloved friends and associates for numerous code reviews and innumerable verbal discussions.

\section{REFERENCES}

[1]. Sidhant Gupta, Dan Morris, Shwetak N Patel, Desney Tan, SoundWave: Using the Doppler Effect to Sense Gestures, ACM, 2012

[2]. Kaustubh Kalgaonkar, Bhiksha Raj, One-handed gesture recognition using ultrasonic sonar, IEEE, 2009

[3]. Thomas Schlomer, Benjamin Poppinga, Niels Henze, Susanne Boll, Gesture Recognition with a Wii Controller, Proceedings of the 2nd international Conference on Tangible and Embedded interaction, 2008

[4]. Afshin Sepehri, Yaser Yacoob, Larry S. Davis "Employing the Hand as an Interface Device", Journal of Multimedia,

[5]. Lars Bretzner and Tony Lindeberg "Use Your Hand as a 3-D Mouse ...", Proc. 5th European Conference on Computer Vision (H. Burkhardt and B. Neumann, eds.), June 1998.

[6]. Matthew Turk and Mathias Kölsch, "Perceptual Interfaces", University of California, Santa Barbara UCSB Technical Report 2003-33

[7]. M Porta "Vision-based user interfaces: methods and applications", International Journal of Human-Computer Studies, 2002.

[8]. Henriksen, K. Sporring, J. Hornbaek, K. " Virtual trackballs revisited", IEEE Transactions on Visualization and Computer Graphics, 2004

[9]. Do Jun-Hyeong, Jung Jin-Woo, Sung hoon Jung, Jang Hyoyoung, Bien Zeungnam, Advanced soft remote control system using hand gesture, Mexican International Conference on Artificial Intelligence, 2006

[10]. K. Ouchi, N. Esaka, Y. Tamura, M. Hirahara, M. Doi, Magic Wand: an intuitive gesture remote control for home appliances, International Conference on Active Media Technology, AMT 2005

[11]. Lars Bretzner, Ivan Laptev, Tony Lindeberg, Sören Lenman, Yngve Sundblad "A Prototype System for Computer Vision Based Human Computer Interaction", 2001.

\section{BIOGRAPHIES}

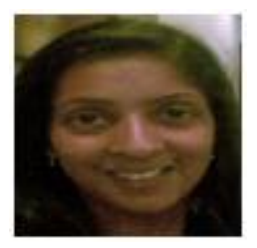

Mrs. Nidhi Gupta is a student of M.Tech (Embedded Systems) at ITM university. She is a Bachelor in Information Technology from University Of Delhi. She holds six years of experience in telecom industry.

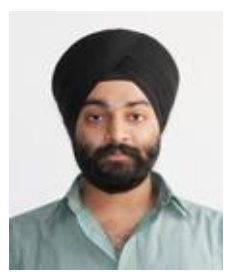

Ramandeep Singh is an Assistant Professor in EECE Department of ITM University, Gurgaon. He is pursuing Ph.D in embedded systems from ITM University. In 2009 he has completed M.E. in embedded systems. He is a B. Tech. graduate from GGSIPU, Delhi. His core research areas are low power embedded systems, robotics, FPGA based embedded systems and SCADA. Prior to joining ITM University he has worked with NXP Semiconductors, Bangalore. He has various publications in international journals and conferences.

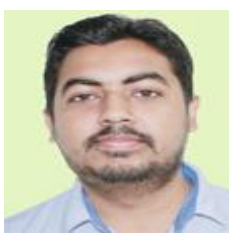

Mr. Sidharth Bhatia expertize is in the domain of Computer Vision and Applications of Robotics. $\mathrm{He}$ has published three research papers in the field of Computer Vision in international journals. He has done his M.Tech (Embedded Systems) from SRM-IST, Chennai and B.Tech from SRM-IST, Modinagar. 